
cultures

Les cahiers de l'Acedle

\title{
Situer la didactique dans le processus de conception de ressources pédagogiques sur support numérique
}

\section{Françoise Demaizière}

\section{(2) OpenEdition}

1 Journals

Édition électronique

URL : http://journals.openedition.org/rdlc/5263

DOI : $10.4000 /$ rdlc.5263

ISSN : 1958-5772

Éditeur

ACEDLE

\section{Référence électronique}

Françoise Demaizière, «Situer la didactique dans le processus de conception de ressources pédagogiques sur support numérique », Recherches en didactique des langues et des cultures [En ligne], 1 | 2004, mis en ligne le 15 novembre 2004, consulté le 11 septembre 2019. URL : http:// journals.openedition.org/rdlc/5263 ; DOI : 10.4000/rdlc.5263

Ce document a été généré automatiquement le 11 septembre 2019.

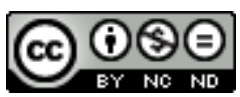

Recherches en didactique des langues et des cultures is licensed under a Creative Commons AttributionNonCommercial-NoDerivatives 4.0 International License 


\title{
Situer la didactique dans le processus de conception de ressources pédagogiques sur support numérique
}

\author{
Françoise Demaizière
}

\section{Préambule, d'un titre à un autre}

1 Le premier titre envisagé pour cette contribution était le suivant "Créer des matériaux pédagogiques sur support numérique - État de l'art (recherche d'un art?)". Je crois que même en modulant l'annonce comme je l'avais fait, un état de l'art est de fait inenvisageable, eu égard à l'immense variété des situations et des points d'entrée des chercheurs, et surtout des praticiens, travaillant dans le domaine. Tricot \& Plégat-Soutjis (2003) relèvent 8348 documents concernant l'ingénierie pédagogique dans la base documentaire Eric (2004), et 40 ouvrages sur le thème chez Amazon, librairie en ligne. Ils trouvent encore 945 documents dans Eric en se restreignant au secteur de la formation à distance. "Comment oser écrire un article qui traite d'ingénierie pédagogique aujourd'hui ?", se demandent-ils. Ils répondent fort bien à la question en proposant des éléments au débat et aux recherches et développements en termes d'ergonomie et de psychologie des apprentissages. J'essaierai donc de répondre en partie à ma question première ("recherche d'un art ?") par le biais d'analyses considérant la place de la didactique. Dans le même article, Tricot et Plégat-Soutjis nous indiquent qu'ils ont souvent rencontré "des équipes travaillant sans méthode explicite". Nous serons d'accord ici, je pense, pour constater que le cas est courant, peut-être plus particulièrement en France comme le suggère l'article. Nous sommes en recherche de moyens plus efficaces et adaptés..., prenant en compte les exigences scientifiques et didactiques universitaires, et de moyens pour faire prendre conscience que la production de ressources nécessite des formes d'organisation. $\mathrm{J}$ 'essaierai d'apporter quelques éléments à la recherche de cet art difficile. Art, artisanat 
ou industrie d'ailleurs? Il n'est pas inintéressant de poser la question. J'évoquerai divers exemples ou cas de figure qui la suggèrent. D'un titre à un autre, $j$ 'essaierai donc ici de poser quelques jalons pour une prise en compte d'éléments pertinents pour que les didacticiens situent mieux leur discipline et que les concepteurs (qui peuvent être les mêmes personnes, chercheurs et praticiens, développeurs, chefs de projet...) trouvent quelques points de référence pertinents. Je serai influencée dans mes points d'entrée et mes exemples de référence par mes expériences de didacticienne et de responsable d'un diplôme de chef de projet multimédia pour la formation (Demaizière \& Cord-Maunoury, 2003).

\section{Matériaux ou ressources pédagogiques, quelle actualité ?}

2 On parle souvent aujourd'hui de "ressources" plutôt que de "matériaux" (Develotte \& Pothier, 2004), ce qui élargit le champ couvert. Les organisatrices de la journée d'étude ont souhaité cerner plus précisément le propos en parlant de "matériaux pédagogiques". La précaution était sans aucun doute utile si l'on regarde la variété des acceptions du terme ressources. Dans Algora en ligne du mardi 20 avril dernier, je relève ceci :

lorsque l'on évoquait il y a quelques années la question des freins au développement des dispositifs de formation ouverts et à distance (...). Lorsqu'on pose la même question aujourd'hui, l'accusation se porte de plus en plus fréquemment, d'une part, sur les prescripteurs, d'autre part, sur la question des ressources (Collectif, 2004).

3 Sans que l'on sache toujours très bien, d'ailleurs, de quoi il est précisément question, (s'agit-il de ressources financières, humaines, matérielles, pédagogiques, etc.), lesdites ressources sont désormais au cœur du débat sur la mise en oeuvre de dispositifs innovants : où les trouver, faut-il les produire, doit-on les partager, doit-on les acheter, puis-je les voler (...), combien ça coûte, comment ça marche, est-ce efficace, comment les intégrer, (...) autant de questions, autant de doutes, qui témoignent de la dure vie de pionnier en e-formation. Certes, rien de plus normal en période d'innovation mais tout de même, depuis le temps que cela dure, il devient urgent de baliser le terrain si l'on veut aider les pionniers à se faire une idée de la mer avant qu'ils ne l'atteignent.

Ce texte d'Algora présente un dossier sur la production de ressources numériques pour la formation du site ministériel Educnet (Educnet, 2004). A la consultation dudit dossier, on constate que les préoccupations ne sont pas principalement didactiques.

5 Je m'interrogerai donc ici sur les moyens de situer la didactique au sein des activités de production de ressources ou de matériaux pédagogiques.

6 Cette approche est à la fois de pleine actualité, les lignes ci-dessus le rappellent. Elle est en même temps marginale par rapport à un certain nombre de préoccupations tant pédagogiques que didactiques du moment. Ainsi, je considérerai que ne sont pas, du moins pas d'emblée ou seulement partiellement, concernées par nos interrogations des activités comme la cyberenquête, l'exploration de la Toile pour y trouver des documents "authentiques", la mise sur pied d'activités collaboratives au travers de la réalisation de projets gérés sur une plate-forme de FOAD (Formation Ouverte À Distance). Il ne s'agit pas alors de créer des matériaux mais des activités ou des tâches (je n'entrerai pas ici dans les distinctions possibles entre activités et tâches). Certaines de ces activités ne font aucun 
appel à des ressources pédagogiques. Il n'en reste pas moins que la production de ressources ou matériaux reste importante et significative.

7 Un certain nombre de personnes produisent des matériaux pédagogiques, même si leur activité ne fait pas l'objet d'autant de recherches ou de comptes-rendus qu'à d'autres moments de l'histoire des relations entre enseignement / apprentissage des langues et technologies. Cette production existe et elle est centrale pour un certain nombre de dispositifs. Dans le cas d'un travail collaboratif par exemple, on donnera accès ponctuellement à un rappel de références de base, à des exercices, à un mémento grammatical. Le statut "secondaire" de ces ressources ne justifie pas qu'on ne veille pas à leur qualité didactique, en termes d'adéquation scientifique et pédagogique. Elles méritent beaucoup plus d'attention qu'on ne leur en accorde généralement.

Je terminerai ce paragraphe en rappelant que s'il y a aujourd'hui consensus dans le milieu, c'est plutôt pour considérer que la qualité d'ensemble des ressources disponibles est moindre qu'il y a quelques années.

\section{Didactique et conception de ressources numériques}

Le point d'entrée retenu ici me conduira à relever :

- pour la didactique, la qualité scientifique et pédagogique ;

- pour les ressources numériques, la bonne exploitation des potentialités du support, la bonne adaptation à ses limites, l'ergonomie, l'utilisabilité ;

- pour la conception, les procédures de constitution d'équipes, de répartition des compétences et de conduite de projet.

10 La fiabilité ou la sophistication techniques pourraient constituer pour certains un quatrième volet. Je les ai volontairement écartées car c'est là que peuvent se perdre la didactique ou le concepteur de ressources qui finit par devenir informaticien ou développeur multimédia plus que didacticien ou spécialiste de la bonne utilisation des supports numériques (en fonction d'un projet pédagogique scientifiquement et donc didactiquement étayé) $)^{1}$.

11 Il s'agira bien de voir comment placer la didactique suivant les différents points d'entrée ou cas de figure, sans l'écarteler, en la tirant vers une technicité qui ne relève pas de son champ. Le risque est moindre, à mon sens, de voir le didacticien devenir un spécialiste de la conduite de projet. Les spécialistes de langues semblent plus tentés par le développement informatique que par la conduite de projet ou le management.

Comment situer le pôle pédagogique entre la compétence didactique et la spécialisation en conception de ressources numériques? J'ai indiqué, en première approximation, ce pôle du côté de la didactique, mais tout pédagogue est-il didacticien? La question est à entendre ainsi: tout pédagogue ou formateur possède-t-il dans son répertoire de compétences ou d'habitudes professionnelles, dans son expertise de praticien, une disposition à la réflexivité sur les pratiques, une habitude de prise de recul pour situer ses actions par rapport à l'ensemble des pratiques et méthodologies du domaine, une habitude de retour vers les domaines ou les disciplines de référence pour vérifier l'adéquation de ses choix ? Ce lien avec la pédagogie est-il à rechercher plutôt du côté du spécialiste des technologies pour la formation, par exemple le chef de projet multimédia (voir plus loin, paragraphe 5.5.)? 
13 Autour des positionnements évoqués ci-dessus se dessinent aujourd'hui un certain nombre d'enjeux importants pour nos disciplines ou nos spécialisations. Je vais donc essayer de clarifier le propos. Pour cela il convient d'abord de balayer la diversité des cas de figure possibles. Je pointerai ensuite quelques évolutions récentes significatives à mon sens en commentant quelques exemples représentatifs.

\section{Diversité des cas de figure}

Dans quels cadres se conçoivent des ressources pédagogiques numériques? J'évoquerai quelques cas de figure.

\subsection{Recherche développement dans le cadre d'une recherche universitaire en didactique des langues}

15 Il pourra s'agir d'une recherche menée dans le cadre de la réalisation d'une thèse par exemple. Le point fort sera logiquement la recherche didactique ; l'expertise en matière de conception de ressources numériques ou de conduite de projet variera grandement en fonction de l'environnement du chercheur et de son expérience préalable.

Dans le cas le plus favorable, on aboutit ici au produit "rêvé" : recherche didactique et développement du produit, surtout si le produit est ensuite diffusé largement. Bien évidemment le rêve est rarement réalité et surtout constitue une réalité difficile à reproduire. Le meilleur moyen de tirer profit d'une thèse pour poursuivre sa carrière n'est pas a priori de continuer à produire des ressources en prenant le temps de la recherche préalable.

Dans les cas peu favorables, on peut se trouver ici face à un plus ou moins grand degré d'artisanat ou d'amateurisme en matière de conception, selon l'environnement, le profil du directeur de thèse...

\subsection{Recherche développement dans le cadre d'une recherche universitaire en informatique (EIAH, Environnements Informatiques d'Apprentissage Humain)}

18 On est ici souvent dans le domaine de produits incluant des formes de traitement automatique de la langue ou d'intelligence artificielle.

19 L'aspect didactique des langues pourra être un peu à l'arrière-plan par rapport aux aspects informatiques. Ce qui relève de l'ergonomie ou de l'utilisabilité ne sera pas nécessairement pris en considération prioritairement non plus.

D'une manière générale, on peut dire que bon nombre de produits liés à des recherches universitaires servent de support riche à des expérimentations qui débouchent sur des publications en didactique ou informatique et alimentent la réflexion à l'intérieur de la communauté des chercheurs mais cela sans pour autant déboucher sur des utilisations massives par les apprenants car les critères d'attractivité, de facilité pour se procurer le produit... ne semblent pas atteints auprès des acheteurs ou utilisateurs potentiels. Régulièrement les ressources en restent au niveau du prototype et ne sont guère utilisées 
avec des apprenants en dehors des expérimentations menées pour la recherche ellemême.

21 La recherche est souvent renvoyée à un artisanat interne. La recherche développement a du mal à passer du développement du prototype à une circulation large. L'université n'est pas une firme automobile!

\subsection{Recherche universitaire préparatoire}

22 C'est le cas où la recherche en didactique ne peut aller jusqu'au développement même d'un prototype. Elle en reste aux étapes préliminaires. Et parfois, ceux qui connaissent cette recherche gardent le regret de ne pouvoir voir le produit qui reste virtuel dans le sens originel du terme. Dégager les moyens d'un développement tout simplement correct lorsque l'on est chercheur en didactique n'est pas aisé. Le cadre de Lettres et Sciences Humaines dans lequel on se trouve ne facilite guère les choses.

\subsection{Développement dans le cadre de financements européens ou ministériels}

Des financements importants peuvent être alors mobilisés. L'accent est en général mis sur le développement d'un produit (un prototype ?) qui doit être fourni dans les délais. Quand ces projets sont internationaux, ils mobilisent souvent un industriel qui participe à la fabrication du produit et permet de le diffuser. Quand ce n'est pas le cas, on peut se retrouver dans le cas du prototype évoqué ci-dessus et il peut être très difficile de trouver aussi bien le financement complémentaire permettant de finaliser le produit pour pouvoir le diffuser que le temps à consacrer à une tâche qui ne sera pas valorisée du point de vue de la recherche (ni financée). S'agissant d'actions ministérielles, on demande souvent des partenariats entre établissements dans le souci de prévoir une mutualisation des ressources. Une fois les produits achevés, le problème est parfois d'obtenir un réel échange. On se heurte au phénomène bien connu de méfiance / défiance vis-à-vis d'une réalisation venant de l'extérieur (voir paragraphe 4.8.). Le problème est persistant. Or tant que l'on n'arrivera pas à faire circuler les produits hors de leur cadre de production, il sera impossible ou déraisonnable de mobiliser les moyens conséquents qui sont pourtant nécessaires à une production multimédia.

\subsection{Création de ressources pour un client extérieur par un laboratoire ou un centre universitaire}

24 Je pense à des travaux tels que ceux décrits dans Depover et al. (2000). Il s'agit, en l'occurrence, d'un centre de recherche en sciences de l'éducation. Peut-on envisager des actions de "service" similaires pour des centres de langues? S'agira-t-il alors de centres de recherche sur la didactique des langues ? À mon avis plus difficilement.

Il me semble que l'on trouve plus aisément une prestation de chercheurs tournée vers l'extérieur dans des domaines généralistes comme l'informatique ou les sciences de l'éducation que dans le cas de la recherche en didactique des langues. On touche à un point important pour notre questionnement : la recherche en didactique des langues se tourne finalement relativement rarement vers des activités de production, d'où un 
certain nombre de décalages que l'on constate entre les caractéristiques des ressources proposées sur le marché et les résultats et préconisations de la recherche.

On peut constater également que la littérature de recherche est peu fournie en didactique dans le domaine par rapport aux chiffres évoqués dans l'article que je citais au paragraphe 1 (Tricot \& Plégat-Soutjis, 2003). Il ne me semble pas y avoir, en didactique des langues, de documents concernant la création de ressources pédagogiques qui soient largement reconnus comme références incontournables par le milieu.

\subsection{La création par un éditeur privé de produits de formation multimédia}

On pourrait penser à une comparaison avec le marché du livre scolaire. Il y a des similitudes mais on remarquera que le poids de l'inspection ne sera pas exactement le même, par exemple, dans la mesure où ici on ne va pas entrer dans le cadre des manuels achetés par les établissements. Le label RIP (Reconnu d'Intérêt Pédagogique) du Ministère fonctionne toutefois sur un mode un peu similaire. Cette comparaison avec le monde scolaire ne vaut évidemment que pour certains produits. Pour la formation d'adultes, qu'il s'agisse de formation initiale ou continue, la situation est différente et les fournisseurs de produits ne seront en général pas les mêmes. Toutefois on retrouvera souvent le principe de l'auteur qui propose son idée ou son produit à un éditeur qui le diffuse. L'activité de création de ressources est une activité annexe par rapport à une activité de formateur ou d'enseignant.

\subsection{La création dans une perspective industrielle, l'entreprise de création de ressources}

J'établis ici une catégorie spécifique pour le cas où, dans le secteur privé, ce sont des professionnels de la création de ressources de formation qui vont concevoir la ressource. On bascule vers une situation où des concepteurs professionnels, sans spécialisation en langues, vont éventuellement faire appel ponctuellement à des formateurs ou didacticiens, sans que ces derniers soient auteurs / concepteurs. Leur rôle est un rôle d'expertise, de conseil. Ils ne pilotent pas la conception. Je vais y revenir (voir paragraphes 6.1. et 6.2.).

\subsection{Création par un enseignant ou un groupe d'enseignants travaillant en interne pour leurs propres apprenants}

C'est un cas de figure classique. Il existe une longue tradition du désir de créer de "petits" produits adaptés à son institution, ses apprenants. Le syndrome du "not invented here", la méfiance envers tout ce qui n'est pas maison, qui est fermé, parachuté de l'extérieur... sont bien répertoriés (voir Naymark, 1999 : 41, par exemple). Nombre de formateurs ont une méfiance instinctive envers ce qui n'est pas fait par eux, à leur manière. La peur du "gros" logiciel "fermé" se grossit sans doute du courant qui pousse les enseignants à s'éloigner des manuels depuis quelques années. On ajoutera l'effet laboratoire de langue qui, dans notre communauté, a un impact non négligeable. Les formateurs en langues ont une longue habitude de créer leurs propres exercices sur ou pour des supports techniques qui leur appartiennent en propre: le laboratoire de langue est le domaine réservé des 
spécialistes en langues. La question est aujourd'hui : cet artisanat interne est-il raisonnable à l'heure du multimédia (voir ci-dessus) ?²

\subsection{Premières conclusions - artisanat, travail d'équipe, industrialisation}

On voit qu'existent des projets individuels, pilotés par la recherche ou le désir de création, projets artisanaux, alors qu'ailleurs se développent des formes de conception plus organisées industriellement (au sens large du terme) à partir d'une conduite de projet de conception. Ces dernières sont plus rarement pilotées par la recherche didactique. Si recherche il y a aujourd'hui, elle est plutôt généraliste que disciplinaire, comme indiqué ci-dessus.

L'artisanat assure évidemment de bonnes garanties quant à la cohérence d'ensemble de la production, mais n'est-il pas mission impossible dans le domaine qui nous occupe ici ? Le chercheur peut-il devenir développeur, graphiste...? Le formateur passionné de développement et de plates-formes peut-il garder la didactique à l'esprit? Parfois le responsable, éditeur par exemple, pourra penser que le fait d'avoir un formateur agrégé de langue dans son équipe suffit à lui donner la garantie de qualité scientifique. Il pourra aussi normalement penser que l'important est que cela plaise et se vende. La société de service qui répond à la commande d'un client particulier a des contraintes plus fortes. Le client doit être satisfait du produit qu'il a demandé en fonction de critères plus exigeants que ceux des parents qui achètent un produit ludo-éducatif ou parascolaire pour leur enfant. Néanmoins on a rarement affaire à une exigence didactique relevant de la recherche universitaire. L'adéquation se jouera à un autre niveau.

L'entrée par une pédagogie qui ne prend pas le temps de s'appuyer sur la didactique ou par une pseudo-spécialisation technique occulte ou néglige la dimension didactique, y compris chez des personnes dont le métier est d'enseigner les langues. Les approches plus industrielles, celles où des personnes consacrent une partie importante, sinon la majeure partie de leur temps de travail, à une spécialisation en conception de ressources (dans un aspect ou un autre) donnent des garanties de professionnalisme et d'efficacité dans la conduite de projet mais la didactique des langues n'y trouve pas toujours son compte non plus. Notre problème ici est justement d'essayer de voir où et comment cette didactique peut se trouver exploitée et / ou satisfaite. Où est-elle le moins malmenée ou ignorée ? Où doit-elle essayer de se faire sa place ? On ne peut manquer ici de remarquer que le simple fait que la majeure partie des didacticiens se vivent comme des chercheurs peu concernés par une implication dans des développements est déjà une explication.

\section{Quelques exemples et questions vives pour éclairer le débat}

\subsection{Les "knowledge factories" d'IBM}

Je choisirai de commencer par un exemple a priori très éloigné de nos problématiques universitaires, en évoquant ce qui se passe actuellement chez IBM ${ }^{3}$. J'y trouve en effet trace de mouvements et d'approches pertinents pour mon propos. 
IBM se positionne actuellement d'abord comme une société de service, il est devenu le plus gros consultant mondial. C'est le B (business) du sigle qui est mis en avant plus que le M (machines). Le "e-learning" couvre actuellement $48 \%$ des besoins de formation internes; les chiffres correspondant à la formation sont désormais inscrits dans les résultats de l'entreprise présentés aux actionnaires (un milliard de dollars d'investissement par an pour la formation).

De manière à industrialiser la production des contenus de formation, un réseau de knowledge factories (usines de / à connaissances ?) a été créé. Ce réseau est présent dans 15 pays, dont la France. Il emploie plus de 3000 professionnels, regroupés en équipes d'une trentaine de personnes chacune. On relève les métiers suivants: concepteurs pédagogiques, concepteurs ergonomes, directeur artistique, coordinateur multimédia, développeur intégrateur, chef de projet. Ce qui me parait particulièrement intéressant à relever dans la "logique de production industrielle" qui est mise en avant est la distinction entre les intervenants suivants: expert du sujet, analyste de contenu, concepteur pédagogique, rédacteur.

L'expert de contenu est, évidemment, extérieur à "l'usine", il ne fait pas partie de la ligne de production. À l'intérieur de la chaîne de conception pédagogique, on spécialise les tâches avec un analyste qui rappelle clairement les ingénieurs extracteurs de connaissances qui travaillent pour la mise au point de systèmes experts informatiques (diagnostic médical...) ou les ingénieurs du management des savoirs (voir ci-dessous). On trouve ensuite un concepteur pédagogique qui donne sa forme au scénario pédagogique sans pour autant toujours le rédiger lui-même. Intervient ensuite la phase de rédaction: les rédacteurs vont formuler les consignes, les activités... (et pourront le faire simultanément en plusieurs langues). Ils veillent à la bonne structuration de l'écrit, phrases courtes..., vérifient que le même terme a toujours le même sens, décident si l'apprenant sera vouvoyé ou tutoyé...

L'approche est une approche "en cascades" qui distingue :

- le design : analyse de contenu puis conception détaillée ;

- le développement : rédaction, validation, production des médias ;

- la recette : test d'intégration, tests utilisateurs.

38 Cette approche permet de faire progresser en parallèle plusieurs projets. Pendant qu'un premier projet en est à la phase de production des médias, un second pourra en être aux phases d'analyse de contenu, etc.

39 Je relève ici que le spécialiste de la discipline (ou de la technique, un des exemples proposé par IBM concerne la fabrication du pain) est un expert de contenu extérieur à la chaîne de conception pédagogique et que l'expertise pédagogique est une expertise proprement multimédia. Les concepteurs et les rédacteurs sont des spécialistes du "elearning". On peut supposer qu'ils ont eu une expérience antérieure de formateurs mais ils ont un métier "e-learning" défini en tant que tel.

Où se situerait pour nous l'expertise didactique nécessaire à la conception? À mon sens, entre l'expert de contenu, l'analyste de contenu et le concepteur pédagogique. On peut penser que dans beaucoup de cas le didacticien serait compétent comme expert de contenu. Dans d'autres, irait-il consulter des disciplines contributoires pour affermir son propos? Le contraste entre les exigences de contenu, d'exactitude scientifique ne sont pas les mêmes pour Carrefour (exemple évoqué par IBM) et pour l'université. Un didacticien n'acceptera pas qu'une ressource pédagogique se présentant comme 
universitaire soit par certains aspects "incorrecte". Je pense, par exemple, aux remarques annexes que l'on fait parfois dans une activité ou un produit. Une remarque sur la prononciation ou la prosodie non validée auprès d'un spécialiste de phonétique, un mémento grammatical ajouté un peu rapidement à un produit principalement centré sur d'autres aspects et qui sera considéré comme inacceptable par un linguiste (voir les remarques de la fin du paragraphe 2).

\subsection{KM et e-learning}

41 Ce premier exemple pris chez IBM me conduit à dire quelques mots sur des évolutions récentes du monde de la formation en entreprise. On voit en effet des rapprochements s'effectuer entre les procédures du KM (Knowledge Management, gestion ou management des savoirs ou des connaissances, voir Tarondeau, 1998) et le e-learning ou la FOAD (voir Blandin et al., 2004, le livre blanc du FFOD, Forum Français pour la formation Ouverte et à Distance). Le mouvement apparaît également dans les universités, en particulier au niveau de masters professionnels. Chez IBM justement, le saut est déjà fait et une seule direction, plus large que l'ancienne DRH (Direction des Ressources Humaines), regroupe la formation et le KM, avec un intitulé "Human Capital Management".

Le livre blanc du FFOD propose des rapprochements intéressants. Le gestionnaire de connaissances de l'entreprise, dans la perspective du KM, a une fonction proche de celle du directeur de la formation ou du DRH, l'analyste des connaissances se rapproche de l'expert du domaine en formation, l'animateur de communautés de pratiques peut être mis en regard du tuteur qui accompagne l'apprenant en formation, le propriétaire de connaissances ou expert se retrouve dans l'expert consulté pour construire une ressource de formation, l'ingénieur des connaissances dans le concepteur de ressources (Blandin et al., $2004: 15)$.

Le point de convergence vient en partie de ce que FAD (Formation à Distance) et KM utilisent des supports TIC et des approches relevant de l'information / communication pour se déployer, mais le phénomène est plus profond. "Le partage (...) de l'information, de l'expertise, de l'expérience est le postulat de base, l'alpha et l'oméga des technologies et méthodologies du KM" écrit Naymark (2004: 581), dans son analyse du livre blanc du FFOD. Je souhaiterais, en écho, attirer l'attention sur un certain nombre de points qui se retrouvent dans les approches actuelles du travail collaboratif et de la formation à distance ou en non-présentiel appuyée sur les TIC. Je pense à la réflexivité partout mise en avant, nécessaire à un apprentissage efficace et autonome ou autonomisant (en FOAD en particulier), au partage des expériences entre pairs pour arriver à la construction de la connaissance, à l'idée de création de microcultures avec leurs espaces de métaréflexion (voir, par exemple, Henri \& Lundgren-Cayrol, 2001, ou Spinelli \& Dolci, 2004).

\subsection{Des dispositifs de FAD plus universitaires}

Les exemples ci-dessus montrent un positionnement spécifique de différents types d'expertises et de compétences dans l'entreprise: expert de contenu, analyste de contenu, créateur de ressources, animateur / accompagnateur. L'expert est un "sachant" (pour reprendre le jargon du milieu du KM) de haut niveau à qui on ne demande pas de formuler une réponse pédagogique. Cette mise en forme relève, c'est explicitement formulé ainsi chez IBM, de la compétence du concepteur pédagogique, créateur des 
ressources. L'exemple d'IBM représente une forme très aboutie de la division des tâches mais il faut savoir que les dichotomies que j'ai soulignées sont celles de l'ensemble des entreprises créant des supports de formation TIC.

Il est maintenant intéressant de voir que ces positionnements ne sont pas propres à l'industrialisation dans une structure d'entreprise. Si l'on observe ce qui se passe dans certains dispositifs de FOAD universitaires, on constate des phénomènes tout à fait similaires en termes de dichotomie entre expert de contenu et intervention pédagogique. Un certain nombre de dispositifs prévoient ainsi de confier l'accompagnement à un spécialiste de cette fonction qui ne se présente pas comme spécialiste du domaine et ira éventuellement consulter l'expert si une question à laquelle il ne peut répondre surgit au cours de la formation. L'animateur de forum sur une plate- forme de FOAD ne se présente pas toujours comme un expert du domaine (voir, par exemple, les exemples et explications données par les chercheurs du Cueep ${ }^{5}$ dans les Cahiers du Cueep (D'Halluin, 2001).

\section{4. Ébauche d'un éclatement des couples traditionnels de la didactique des langues, d'un élargissement des perspectives ?}

46 Ces choix peuvent faire sursauter notre fibre de didacticiens de la discipline. Comment encadrer des apprenants en langues sans connaître les spécificités de ladite discipline? Comment repérer des erreurs de méthode graves pour la suite si on ne maîtrise pas le domaine scientifique et les problèmes d'appropriation qu'il présente pour les apprenants? Mais, peut-on répondre, est-il bien raisonnable de confier l'animation de forums de FOAD à des enseignants si rigidement enfermés dans leurs postures magistrales habituelles qu'ils ne vont pas savoir s'adapter à une situation nouvelle pour eux et qui demande, pour dynamiser le parcours des apprenants, des attitudes fort différentes de celles qui leur sont habituelles? Certains collègues responsables de dispositifs de FOAD ont fait leurs choix en privilégiant les qualités d'animation ou d'écoute des apprenants. Il s'agit en général de choix faits plutôt à l'initiative de spécialistes en sciences de l'éducation (c'est le cas du Cuepp, évoqué ci-dessus, qui s'appuie sur le laboratoire de sciences de l'éducation Trigone) et pour des matières ou des domaines qui ne sont pas très spécialisés (DAEU - Diplôme d'Accès aux Études Universitaires, par exemple).

47 Ces exemples qui reconnaissent de plein droit la qualité spécifique du spécialiste des TIC ou de la FOAD me semblent intéressants. Ne faut-il pas s'interroger de manière similaire pour la création de ressources pédagogiques adaptées aux supports TIC? Un formateur est-il toujours le plus apte à construire un scénario TIC? S'il n'a pas de compétence didactique fortement affirmée ni de spécialisation en TIC pour la formation, ne risque-ton pas de voir des approches cherchant à reproduire des situations de classe inadaptées au support? Ne risque-t-on pas de s'en tenir à quelques interactions limitées entre apprenant et logiciel par manque de connaissance de ce qui peut se faire?

La didactique des langues me semble avoir encore trop de mal à s'extraire de ses cadres habituels. En premier lieu, le cadre "classe de langue", qui ignore les utilisations individuelles de ressources TIC, les centres de ressources, la distance; en second lieu, le couple formé par le didacticien (ou la didactique) et l'enseignant (le formateur), couple dans lequel la pédagogie est $\mathrm{du}$ ressort du praticien enseignant. Ce couple est-il indissoluble ou doit-il évoluer? Doit-il au moins être considéré dans d'autres dimensions? Les exemples que j'ai évoqués ci-dessus me semblent aller dans le sens d'un 
réexamen des équilibres et des définitions des différents rôles. C'est pourquoi je souhaiterais revenir sur la spécialisation en TIC pour la formation et évoquer maintenant en particulier le chef de projet multimédia.

\subsection{Le chef de projet multimédia}

49 Ce nouveau métier commence à apparaître, il existe des diplômes y préparant. Le chef de projet est (dans le cas idéal évidemment, pas dans les circonstances où l'on considérera qu'il est un spécialiste de la médiatisation ou du développement, ou un informaticien) un spécialiste de l'utilisation des TIC pour l'éducation et la formation. Par sa culture professionnelle il connaît les différentes mises en œuvre possibles. Pour ce qui nous intéresse ici, il connait donc les différents types de ressources qui peuvent (ou ont pu) exister ${ }^{6}$ ainsi que leur plus ou moins bonne adaptation à tel ou tel type de support ou de dispositif. Il a une compétence pour scénariser un contenu sur les supports TIC et pour opérer une conduite de projet efficace. Il pourra dialoguer avec les développeurs ou ceux qui vont proposer telle ou telle solution technique en gardant à l'esprit l'importance des choix didactiques, méthodologiques et pédagogiques.

En milieu universitaire ce chef de projet se présentera éventuellement avec un profil d'ingénieur d'étude ou de recherche (on recrute actuellement des "ingénieurs pédagogiques" dans diverses universités) plutôt que d'enseignant chercheur. On voit bien qu'en ce cas, son intégration dans la communauté pourra causer quelques difficultés : on le verra plus facilement comme aide technique que comme pilote du projet.

\section{Quelles combinaisons d'expertises ou de spécialisations envisager?}

51 Examinons donc différents cas de collaborations envisageables telles qu'elles se rencontrent ou pourraient être envisagées. Il conviendra pour chacune de se demander où se situera la responsabilité du projet, qui décidera en cas de désaccord, qui consacrera une part importante de son temps de travail au projet et qui sera un collaborateur occasionnel.

\subsection{Un spécialiste du scénario multimédia assisté d'un expert de contenu}

C'est un cas courant dans les développements industriels ou très spécialisés. Comme pour la construction d'un système expert ou dans les procédures de KM, on va extraire les connaissances de l'expert par une collaboration ponctuelle compatible avec les obligations d'un expert qui, par définition, n'est pas ici un spécialiste de la formation, avec ou sans TIC.

Cet expert est-il pour nous un didacticien "généraliste" ou un spécialiste de phonétique, d'anthropologie culturelle...? 


\subsection{Un spécialiste multimédia assisté d'un formateur considéré comme expert de contenu}

On considère que le formateur est expert du contenu. Il sera parfois plus aisé d'obtenir une participation conséquente de ce formateur au projet que dans le cas d'une personne considérée comme expert de haut niveau consultable ponctuellement uniquement. Quelques inconvénients potentiels ont été signalés ci-dessus.

\subsection{Un didacticien assisté d'un développeur}

C'est le cas de la recherche pour une thèse, évoqué plus haut. Ce travail est plus difficile à reconnaître dans le calcul du service de l'enseignant chercheur et donc à pérenniser. Ce devrait être un champ d'action privilégié pour l'enseignant chercheur qui fait sa recherche en didactique. Si le didacticien est isolé, il y a un risque évident de manquer de moyens techniques pour la réalisation. On peut penser qu'il n'y a alors plus besoin du spécialiste du scénario TIC, mais comment gérer la conduite de projet et assurer le bon avancement du projet et la coordination avec l'équipe de développement?

\subsection{Une solution optimale : didacticien et spécialiste TIC en collaboration?}

Cette abondance de compétences est-elle à rechercher? A priori elle semble séduisante. Toutefois on se rappellera que les spécialistes de l'extraction de connaissances pour la construction de systèmes experts, par exemple, soulignent qu'il ne faut pas mettre en concurrence plusieurs experts car l'on risque alors de ne pas trouver de ligne directrice cohérente au produit. Il peut y avoir un effet perturbateur du choc entre deux approches spécialisées, efficaces isolément mais difficiles à conjuguer au sein d'une production unique.

Une solution idéale serait-elle une université où existerait un service multimédia pour la formation qui pourrait prendre en charge la production dans le cas où les experts universitaires ne seraient pas disponibles pour le projet TIC et apporter une collaboration plus ponctuelle à ceux qui prennent largement en charge la conduite de leur réalisation mais tireraient néanmoins profit d'un échange avec une ou des personnes plus généralistes qu'eux ? On peut rêver...

\section{Conclusion : solliciter la recherche didactique pour qu'elle intègre dans son champ les métiers du multimédia}

La didactique cherche à faire le lien entre les fondements théoriques ou méthodologiques et les mises en pratique, elle a longtemps considéré plus ou moins consciemment que ces mises en pratique relevaient du métier de formateur dans sa classe. Envisager des situations d'apprentissage appuyées sur les TIC pousse à sortir de ladite classe. Envisager la création de ressources pédagogiques numériques contraint à sortir d'une compétence pratique univoque et fixe de formateur pédagogue spécialiste du présentiel pour 
envisager la ou les spécialisations de conception multimédia ou de conduite de projet multimédia.

Les remarques faites ci-dessus montrent bien que beaucoup de problèmes importants relèvent de la politique universitaire nationale ou locale : création de cellules spécialisées dans les dispositifs multimédia et recrutement de personnels compétents et intégrés à la vie de l'établissement, soutien à des demandes de financement pour des projets d'envergure, reconnaissance "correcte" du temps passé à la création... Cela ne relève pas de la recherche, didactique ou autre. Néanmoins la recherche est dépendante de ces choix, il faut bien le reconnaître.

60 À l'heure où les besoins en formation en langues à l'université explosent avec la mise en place du LMD et le développement de nombreux cursus exigeant un bon niveau en langue étrangère (ingénieurs, masters professionnels...), il est bon de se rappeler que l'enseignement universitaire se doit (se devrait) de s'appuyer sur la recherche. Dans un département de langues pour spécialistes d'autres disciplines, Lansad, ou un centre de langues, cette recherche devrait logiquement être de type didactique. Nous savons qu'il y a loin de la logique à la réalité des choses ; néanmoins, un vaste champ pourrait s'ouvrir. Je continue de souhaiter que la didactique accepte d'être interventionniste et de s'impliquer de manière suivie dans des actions de recherche développement et dans des recherches actions liées à l'usage des TIC et donc en particulier à la création de ressources pédagogiques.

\section{BIBLIOGRAPHIE}

Blandin, B., Bayou, C., Dambach, Y., Engelibert, J., Fage, C., Voyeux, N. (2004). E-Learning et knowledge management : quelle convergence ?. Consulté en septembre 2004. http://www.fffod.org/ $\mathrm{fr} /$ doc/ElearningKM_fffodVFC.doc

Collectif (2004). "Produire et organiser des ressources numériques pour la formation - Éditorial". Algora en ligne, $\mathrm{n}^{\circ} 74,21$ avril. Consulté en avril 2004. http://www.algora.org/fr/index.asp

Demaizière, F. \& Cord-Maunoury, B. (2003). "Penser une formation aux TIC - Une professionnalisation des acteurs de la formation : formateurs et chefs de projet. In Naymark, J. \& Depover, C. (dir.). E-formation, des enjeux aux usages. Distances et savoirs, vol. 1, 4. pp. 533- 550.

Depover, C., Quintin, J.-J. \& De Lièvre, B. (2000). "La conception des environnements d'apprentissage : de la théorie à la pratique / de la pratique à la théorie". Alsic, Apprentissage des Langues et Systèmes d'Information et de Communication, vol. 3, 1. pp. 3-18. Consulté en septembre 2004. http://alsic.u-strasbg.fr/Num5/depover/alsic_n05-rec4.htm

Develotte, C. \& Pothier, M. (dir.) (2004). La notion de ressources à l'heure du numérique. Notions en questions, $\mathrm{n}^{\circ}$ 8. Lyon : ÉNS Éditions.

D'Halluin, C. (coord.) (2001). Les cahiers d'études du Cueep - Usages d'un environnement médiatisé pour l'apprentissage coopératif, $n^{\circ} 43$, janvier 2001 . 
Éducnet (2004). Produire et organiser des ressources numériques pour la formation. Consulté en septembre 2004. http://www.educnet.education.fr/dossier/formation-ressources/default.htm

Floréan, D. \& IBM (2003). Solutions IBM pour l'éducation et la formation. Documents présentés lors d'un exposé.

Henri, F. \& Lundgren-Cayrol, K. (2001). Apprentissage collaboratif à distance. Sainte Foy : Presses de l'Université du Québec.

Naymark, J. (1999). "Typologie des produits et de dispositifs". In Naymark, J. (dir.). Guide du multimédia en formation. Paris : Retz. pp. 36-45.

Naymark, J. (2004). "À propos de la publication du FFOD, 'E-Learning et knowledge management : quelle convergence ?'". Distances et savoirs, vol. 1, 4. pp. 579-581.

Pouts-Lajus, S. \& Riché-Magnier, M. (1998). L'école à l'heure d'Internet - Les enjeux du multimédia dans l'éducation. Paris : Nathan.

Spinelli, B. \& Dolci, R. (2004). "Création d'une culture partagée dans un environnement de communication virtuelle". Présentation proposée à la journée Formations en langues et Internet : quels aspects collaboratifs ?, ÉNS LSH, Lyon, mars 2004. Consulté en septembre 2004. http:// www.inrp.fr/rencontres/seminaires/2004/praxis/Lyon2004_B.ppt

Tarondeau, J.-C. (1998). Le management des savoirs. Paris : PUF.

Tricot, A. \& Plégat-Soutjis, F. (2003). "Pour une approche ergonomique de la conception d'un dispositif de formation à distance utilisant les TIC". Revue Sticef (Sciences et Technologies de l'Information et de la Communication pour l'Éducation et la Formation), vol. 10. Consulté en septembre 2004. http://sticef.univ-lemans.fr/num/vol2003/tricot-07s/sticef_2003_tricot_07s.htm

\section{Sites}

Eric - Education Resources Information Center (2004). Consulté en octobre 2004. http:// eric.ed.gov/

\section{NOTES}

1. Je n'ignore évidemment en aucun cas ces didacticiens qui réussissent à allier compétence de programmation / médiatisation et de conception à leur compétence didactique. Ils sont toutefois rares et me semblent difficilement pouvoir être donnés en exemple même s'ils ont droit à toute mon admiration et si je les envie, moi qui ne peux aller très loin sans une collaboration avec plus technicien(ne) que moi.

2. On rappellera également le décalage entre l'investissement démesuré consenti par des pionniers qui réussissent à mener à bien des expérimentations réussies et ce qui peut raisonnablement être attendu du professionnel "moyen" (raisonnable, souhaitant avoir une vie privée ou investir le temps nécessaire à sa formation de base...) qui est pointé fort à propos dans l'ouvrage de Pouts-Lajus \& Riché-Magnier (1998).

3. Je m'appuie ici sur un exposé de Denis Floréan d'avril 2004 et sur les documents IBM fournis à cette occasion (Floréan \& IBM, 2004).

4. J'introduis des guillemets dans la mesure où IBM continue, avec d'autres, à utiliser cette terminologie devenue plus rare dans les milieux académiques où elle tend à être remplacée par FOAD (Formation Ouverte À Distance). 
5. Le Cueep, Centre Université- Économie d' Éducation Permanente, Université des Sciences et Technologies de Lille, est un centre phare dans le domaine de la formation continue.

6. N'oublions pas qu'un certain nombre de réalisations de qualité ne sont plus consultables aujourd'hui aisément et demandent donc une certaine spécialisation pour être connues par le biais de la littérature en particulier.

\section{RÉSUMÉS}

Cet article propose quelques jalons permettant de situer la didactique dans le processus de conception de ressources pédagogiques numériques. Après avoir évoqué la grande diversité des cas de figure envisageables, de la recherche universitaire à la production industrielle, le problème de la répartition des rôles et des expertises est posé à partir d'exemples. Les apports possibles d'approches souvent peu prises en compte par la communauté des didacticiens des langues sont indiqués. Il est suggéré que la didactique des langues pourrait trouver intérêt à sortir de cadres traditionnels tels que l'association exclusive entre la didactique et la pédagogie (de la classe). Un regard vers certains usages de l'entreprise ou de la formation à distance pousse à reconsidérer les expertises les mieux adaptées à la production de ressources numériques et à envisager l'intérêt de la spécialisation professionnelle en création multimédia en particulier.

The text tries to give a few elements aiming at a more adequate connection between didactics as a research field and the design of multimedia language learning materials. The author stresses the great variety of design teams and environments one can come across and relies on non academic or continuing education perspectives to suggest that language specialists could gain benefit from looking at professional specialities or organizations that are still often unfamiliar to them.

\section{INDEX}

Mots-clés : didactique, conception, ressources pédagogiques, expertises

Keywords : didactics, design, learning materials, expertise

\section{AUTEUR}

\section{FRANÇOISE DEMAIZIÈRE}

Françoise Demaizière travaille depuis de nombreuses années sur le lien entre la didactique des langues et la production de logiciels pédagogiques. Elle intervient tant en formation initiale qu'en formation continue. Université Paris 7, case 7023, 2 place Jussieu, 75005 Paris demaizie@ccr.jussieu.fr http://www.univ-paris7.fr/AEM 